\title{
BILAN DES EFFORTS D'INTRODUCTION DE LA TRUITE BRUNE (SALMO TRUTTA) DANS LES EAUX DE LA PLAINE DE MONTRÉAL, QUÉBEC
}

\author{
P. DUMONT et J.R. MONGEAU
}

Service de l'aménagement et de l'exploitation de la faune
Ministère du Loisir, de la Chasse et de la Pêche
6255, $13^{\circ}$ Avenue - MONTREAL (Québec) CANADA H1X 3E6

\section{RÉSUME}

Introduite en Amérique du Nord en 1883, la truite brune (Salmo trutta) a été ensemencée pour la première fois au Québec en 1890. Ce n'est cependant qu'au début des années 1950 qu'elle a commencé à susciter l'intérêt des aménagistes et des pêcheurs québécois en raison de sa tolérance plus grande que celle des ombles indigènes (Salvelinus $s p p$ ) aux températures élevées et à la compétition interspécifique. Au Québec, elle n'est actuellement implantée que dans la partie sud-ouest du bassin versant du fleuve Saint-Laurent. La découverte, en 1965, de la présence de l'espèce dans les eaux de la plaine de Montréal, considérées comme peu appropriées aux exigences des salmonidés, est à l'origine d'un programme d'ensemencement soutenu dans le fleuve SaintLaurent et dans la partie aval de certains de ses tributaires. En dépit des températures estivales relativement élevées $\left(\max .>25^{\circ} \mathrm{C}\right.$ ) et de la présence de populations de poissons abondantes et diversifiées, la survie de la truite brune dans ces eaux est excellente et sa croissance est rapide. Ses performances à la pêche sportive sont bonnes en termes de succès, de taille et de fréquence des retours des poissons ensemencés. La fraye a été observée en plusieurs occasions. Cependant, l'analyse récente des statistiques de capture d'un groupe de pêcheurs volontaires confirme l'hypothèse d'une contribution significative des ensemencements à la récolte et suggère même la possibilité d'une contribution négligeable de la reproduction naturelle. Les possibilités d'introduction de la truite brune sont limitées par des mesures réglementaires qui visent à protéger l'intégrité des communautés aquatiques établies au nord du $46^{\circ}$ degré de latitude Nord. Toutefois, vu que l'espèce, à l'instar de plusieurs autres salmonidés non indigènes, est déjà présente dans les bassins des Grands Lacs, du fleuve Saint-Laurent et de divers cours d'eau de plusieurs provinces et états de la Côte Atlantique, il paraît probable qu'elle s'établira dans les tributaires de l'estuaire et du Golfe du Saint-Laurent, où elle pourrait éventuellement entrer en interaction avec les peuplements anadromes et dulcicoles de saumon atlantique (Salmo salar), d'omble de fontaine (Salvelinus fontinalis) et d'omble chevalier (Salvelinus salvelinus).

\section{AN EVALUATION OF THE INTRODUCTORY TRIALS OF THE BROWN TROUT (SALMO TRUTTA) IN THE WATERS OF THE MONTRÉAL LOWLANDS, QUÉBEC}

\section{ABSTRACT}

Introduced into North America in 1883, brown trout (Salmo trutta) was planted for the first time in Québec in 1890. However, it is only at the beginning of the 1950s that this species began to arise interest among Québec wildlife managers, biologists and fishermen on account, comparatively to native charrs (Salvelinus $s p p$ ), of its greater tolerance for rather high temperatures and interspecific competition. In 1965, the discovery of this species in the lowlands of the Montreal area, considered of poor interest for salmonids, has been the starting-point of a sustained planting program in the Saint-Laurent river and in the lower reaches of a certain number of its tributaries. In spite of the relatively high summer temperatures (max. $>25^{\circ} \mathrm{C}$ ) and the abundant and diversified fish fauna in these waters, the brown trout thrives successfully and grows rapidly. Its sport fishing yield is satisfactory as to fishing success, size of specimen caught and amount of fish planted returned. Spawning has been observed on many occasions. However, the analysis of recent statistics derived from the catch of a group of devoted fishermen supports the hypothesis of a significant contribution of the plantings to the fishing harvest and, on the other hand, the possibility of an insignificant contribution by natural reproduction. The planting possibilities of the brown trout are limited by measures aiming to protect the integrity of aquatic communities north of the $46^{\circ} \mathrm{N}$. However, on account of the fact that this species, like many other non native salmonids, is already present in many basins like the Great Lakes, the Saint-Laurent river and several watercourses of many provinces and states of the Atlantic Cost, it seems likely that it will eventually establish itself in the Saint-Laurent estuary and Gulf tributaries, where it might interfere with the anadromous and fresh water populations of atlantic salmon (Salmo salar), brook charr (Salvelinus fontinalis) and arctic charr (Salvelinus salvelinus). 


\section{INTRODUCTION}

C'est en 1883 que la truite brune (Salmo trutta) a été introduite en Amérique du Nord. Des œufs, importés d'Allemagne, furent utilisés sur la côte est des États-Unis (MACCRIMMON et MARSHALL, 1968 ; MACCRIMMON et al., 1970). L'introduction de l'espèce au Québec remonte à 1890, alors que des fretins, en provenance de l'État de New York, furent déversés dans le lac Brûlé, à environ $150 \mathrm{~km}$ au nord-ouest de Montréal (LEGENDRE et al., 1980). Toutefois, cette truite ne commença à susciter l'intérêt des pêcheurs et des aménagistes qu'au début des années 1950 . Ceux-ci espéraient alors que, en raison de ses habitudes ichtyophages et de sa tolérance plus grande pour les températures élevées, elle serait en mesure de relancer la pêche dans les eaux fraîches des plateaux montagneux du Québec méridional qui, peu de temps auparavant, étaient peuplées de salmonidés indigènes. En effet, cet habitat de l'omble de fontaine (Salvelinus fontinalis) avait été dégradé, au cours des décennies précédentes, par la déforestation et par l'implantation d'espèces compétitrices, telles la perchaude (Perca flavescens) et différentes espèces de centrarchidés et de cyprinidés, à la faveur du transport et de l'utilisation de poissons-appâts. Les efforts d'introduction se multiplièrent dans la plupart des affluents des deux rives de la rivière des Outaouais et du fleuve Saint-Laurent depuis la frontière de l'Ontario jusqu'à la ville de Québec. La majorité de ces ensemencements, réalisés de façon sporadique et généralement au moyen d'alevins ou de jeunes fretins, ne donnèrent pas les résultats attendus et seules quelques étendues d'eau, ensemencées de façon soutenue, bénéficièrent d'implantation. COTÉ (1983) mentionne sa présence dans tout au plus une trentaine de rivières et de lacs du Québec méridional.

Au début des années 1960 , alors que la ferveur pour la propagation de l'espèce dans les eaux fraîches et oligotrophes s'estompait, survint un fait nouveau et inattendu qui devait relancer tout le processus: des captures de truites brunes dans les eaux eutrophes et relativement chaudes des basses terres de la plaine de Montréal, dans le fleuve Saint-Laurent et dans le cours inférieur de ses tributaires, commençaient à ètre rapportées par les pêcheurs sportifs. Aux yeux de plusieurs observateurs, il paraissait impensable qu'un tel phénomène puisse se produire dans un milieu qualifié à l'époque de "fleuve mort, ruiné par la pollution, l'industrie, la canalisation, etc..." (LEGENDRE et al., 1980).

Suite à cet événement, notre service entreprit, à compter de 1965, un programme d'ensemencements annuels de cette espèce dans les secteurs d'eau vive de la région de Montréal. Bien qu'amorcés sur la base de petites quantités d'alevins, de fretins et d'adultes $(92.850,44.200$ et 1.002 respectivement entre 1965 et 1967), les résultats de ces ensemencements ne se firent pas attendre. Dès 1968, des spécimens de plus de $35 \mathrm{~cm}$ étaient régulièrement rapportés par les pêcheurs (MONGEAU, 1975), si bien qu'entre 1970 et 1975, notre service avait recueilli, sans sollicitation du public, 805 mentions de capture.

Depuis les premiers essais, les pratiques d'ensemencement ont été modifiées. Ainsi, le recours aux fretins a été graduellement abandonné en faveur de poissons d'un an + . Le nombre de sites ensemencés a également été réduit à la lumière des résultats de la pêche sportive et de travaux d'étiquetage, de façon à ne conserver que les plus rentables (en l'occurrence, ceux qui sont caractérisés par des eaux rapides et turbulentes). Actuellement, la plupart des ensemencements sont effectués au cours du mois de mai, en utilisant des spécimens d'un an +. Ces poissons sont les descendants, de première ou de deuxième génération, de géniteurs capturés dans le milieu sauvage, dans deux lacs du sud-est du Québec.

Entre 1983 et 1987, 123.900 truites brunes, représentant environ $20 \%$ de la production gouvernementale de cette espèce, ont été ensemencées dans différents secteurs des cours d'eau de la plaine de Montréal, couvrant ainsi une superficie de 3.260 ha. Les quantités déversées ont cependant été sujettes à des variations annuelles importantes, en raison de problèmes sanitaires éprouvés par les stations piscicoles gouvernementales: elles ont oscillé entre 0 et 48.000 pour des densités annuelles moyennes inférieures à 14,7 individus par hectare de superficie appropriée.

Dans le présent document, nous nous proposons, à l'aide des informations recueillies et compilées par notre service depuis 1965, de tracer un bilan des connaissances et des résultats obtenus au cours de nos efforts d'introduction de l'espèce dans la plaine de Montréal.

\section{TERRITOIRE A L'ETUDE}

Trois cours d'eau, dont les caractéristiques sont nettement différentes, ont reçu la majeure partie des ensemencements. Il s'agit du fleuve Saint-Laurent et de deux tributaires de sa rive sud: la rivière Richelieu, dans sa partie centrale, et la rivière Châteauguay, dans la partie supérieure de son cours (fig. 1).

Au voisinage de Montréal, le fleuve Saint-Laurent forme un important réseau de chenaux et d'îles, qui est le point de rencontre de deux masses d'eau d'origine et de propriétés distinctes. L'une, en provenance du bouclier précambrien, est brune, douce et chargée de matière organique, tandis que l'autre, en provenance des Grands Lacs, est verte, transparente, dure et riche en nutriment. Les 


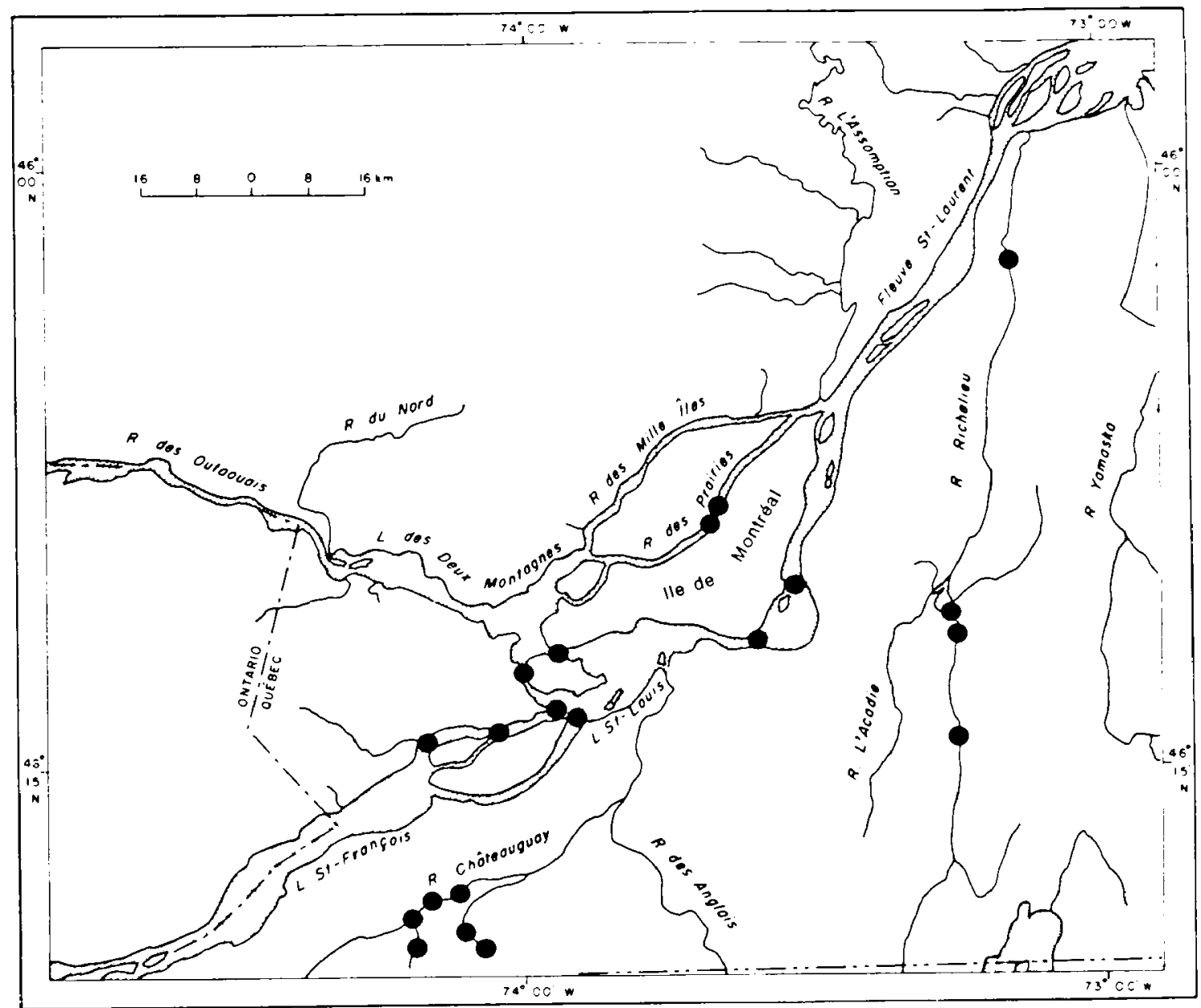

figure 1 : Localisation des sites d'ensemencement de truites brunes dans la plaine de Moniréal. figure 1 : Location of brown trout stocking sites in the Montreal lowlands.

débits impliqués sont très importants : Ia moyenne annuelle est de l'ordre de $8.300 \mathrm{~m}^{3} / \mathrm{s}$ à la hauteur de Montréal. Sauf dans le chenal de navigation, la profondeur est généralement inférieure à $6 \mathrm{~m}$. Le fleuve est couvert de glace en hiver; en période estivale, son eau peut atteindre des températures de l'ordre de $25^{\circ} \mathrm{C}$ et ne présente aucune stratification thermique. Bien qu'affecté par un haut degré de pollution industrielle et domestique, le fleuve demeure productif quant à la vie aquatique en raison de son pouvoir de dilution élevé et de la présence de vastes secteurs de rapides. Environ 90 espèces de poissons d'eau douce et anadromes y ont été répertoriées (MONGEAU et MASSE, 1976).

La rivière Richelieu prend sa source dans un vaste lac oligotrophe, le lac Champlain, avant de drainer une plaine agricole dont les apports sont responsables d'un niveau élevé d'eutrophisation. Ses débits annuels moyens sont de l'ordre de $300 \mathrm{~m}^{3} / \mathrm{s}$ et sa profondeur est généralement in férieure à $7 \mathrm{~m}$. Une quarantaine d'espèces de poissons ont óté recensées dans le secteur ensemencé. Également sous couvert de glace en hiver, l'eau de cette rivière peut atteindre $26^{\circ} \mathrm{C}$ en été et ne manifeste aucune stratification thermique (MONGEAU, 1979).

La rivière Châteauguay, quant au site des ensemencements, est un petit cours d'eau qui prend sa source en milieu montagneux, mi-boisé et mi-agricole, avant de s'écouler dans une plaine agricole. Le secteur ensemencé est caractérisé par des débits faibles $\left(30 \mathrm{~m}^{3} / \mathrm{s}\right)$, une eau de bonne qualité, une profondeur inférieure à $2 \mathrm{~m}$, une température de l'eau inférieure à $25^{\circ} \mathrm{C}$ en été et une faune aquatique composée d'une trentaine d'espèces de poissons (MONGEAU, LECLERC et al., 1979).

Bien que très différents, ces trois cours d'eau présentent des caractéristiques communes : ils sont entrecoupés de secteurs de rapides et connaissent, au cours d'un cycle annuel, des écarts de débits très importants, responsables de crues subites et de grande amplitude au printemps, et, à un degré moindre, à l'automne. 
La pêche récréative revêt une grande importance dans le territoire à l'étude. Une enquête récente révèle qu'en 1985 plus de 193.000 personnes ont consacré environ 2,5 millions de jours à la pêche dans la plaine de Montréal, principalement en vue de la capture de percidés, d'ésocidés et de centrarchidés (ANONYME, 1987). Cette estimation représente environ $15 \%$ de l'effort total de pêche récréative réalisé au Québec.

\section{MATÉRIEL ET MÉtHOdES}

Les résultats qui suivent proviennent de différentes sources. Les paramètres de croissance ont été évalués à l'aide de spécimens capturés lors de travaux d'inventaire ichtyologique ou rapportés à nos laboratoires par des pêcheurs sportifs. L'âge fut déterminè au moyen de l'écaille suivant une méthode décrite par MONGEAU, LEGENDRE et al., (1979). Le coefficient de condition de Fulton a été calculé au moyen de la formule de Tesch (in RICKER, 1971).

Les données de déplacement et de répartition proviennent de deux opérations d'étiquetage: l'une, réalisée de 1968 à 1969 (MONGEAU, 1975), et l'autre, de 1977 à 1981 (MONGEAU et BRISEBOIS, 1982), d'une revue de la littérature, et finalement d'une enquête auprès des Services régionaux d'aménagement et d'exploitation de la faune cuvrant en bordure du fleuve Saint-Laurent. Une partie des résultats ainsi obtenus figure déjà dans DUMONT et al., (1988).

Les observations concernant la reproduction de la truite brune dans la plaine de Montréal proviennent principalement de différents travaux d'inventaire de frayères réalisés dans les eaux vives, à l'occasion d'études d'impacts de projets de développements hydro-électriques (MONGEAU et al., 1980; GUAY et COUILLARD, 1985; ENVIRONNEMENT ILLIMITÉ, 1987 ; BÉRUBÉ, 1983).

La description de l'habitat de la truite brune dans le territoire à l'étude provient d'observations effectuées lors de travaux d'inventaire, d'un exercice de repérage radio-télémétrique dans le fleuve Saint-Laurent (LECLERC, 1984) et de la compilation des déclarations de capture des pêcheurs sportifs.

Enfin, les informations relatives aux particularités de la pêche sportive proviennent d'une campagne de cueillette de statistiques de pêche aux salmonidés dans la région de Montréal. Celle-ci, en vigueur depuis 1983, repose sur la participation d'un groupe de pêcheurs volontaires (de 52 à 70 par année) qui enregistrent, dans un carnet spécialement conçu à cet effet, leur effort de pêche aux salmonidés et leurs captures par espèces et par groupe de taille. Cette campagne vise à suivre l'évolution de la qualité de la pêche ainsi que la progression des saumons non indigènes (Oncorhynchus spp.) dans le couloir fluvial, sans avoir à investir des ressources importantes (DUMONT et al., 1988). Les données recueillies permettent également de vérifier si les poissons ensemencés contribuent effectivement et significativement à la récolte. A cette fin, nous posons l'hypothèse que, si tel est le cas, des variations annuelles des quantités ensemencées se traduisent par des variations parallèles de la contribution des individus de petite taille à la récolte, soit ceux de la catégorie des moins de $30 \mathrm{~cm}$. Par ailleurs, compte tenu de la taille des spécimens ensemencés (11 à $25 \mathrm{~cm}$ ) et des taux de croissance observés dans les eaux de la région, ces captures de petite taille sont susceptibles d'être en majorité des sujets d'un an + . Une description détaillée de la procédure utilisée figure dans DUMONT et DUMAS (1987).

\section{RÉsultats}

\subsection{Habitat}

La compilation des lieux de capture de truites brunes depuis 1965 montre que la plupart proviennent des eaux vives, particulièrement de celles qui coulent en torrents sur des pentes raides, rocheuses, irrégulières, parsemées de grosses roches et de fosses profondes. En saison froide, des captures éparses sont aussi réalisées en eau relativement calme et sur des substrats moins raboteux. Dans l'archipel de Montréal, les concentrations de ce salmonidé sont surtout localisées dans les sites baignés par les eaux vertes, en provenance des Grands lacs, alors que les sites correspondants, en eaux brunes, semblent beaucoup moins favorisés.

LECLERC (1984), interprétant les données de repérage radio-télémétrique ( $\mathrm{n}=89$ ) de 35 truites brunes provenant des rapides de Lachine, à la hauteur de Montréal, caractérise ainsi I'habitat: vitesse de courant supérieure à $0,45 \mathrm{~m} / \mathrm{s}$, profondeur inférieure à $4 \mathrm{~m}$, substrat dur (gravier ou $\mathrm{roc}$ ), présence ou absence de végétation.

\subsection{Déplacements}

En 1968 et 1969, 535 truites brunes âgées de 1,5 à 3,5 ans furent étiquetées et libérees dans le fleuve Saint-Laurent (rapides de Lachine) et dans la rivière Richelieu (aval du barrage de Chambly). Parmi les 36 recaptures rapportées, 33 eurent lieu à l'endroit même de l'ensemencement, une à l'amont, à $24 \mathrm{~km}$, et deux à l'aval, à 16 et $56 \mathrm{~km}$ respectivement (MONGEAU, 1975). 
En 1977, 420 spécimens, de 3 à 5 ans, furentétiquetés et ensemencés dans les deux mêmes sites que les précédents, ainsi que dans la rivière Châteauguay. Les 55 recaptures qui ont été rapportées ont toutes eu lieu à l'endroit même de l'ensemencement ou à proximité (MONGEAU et BRISEBOIS, 1982).

DUMONT et al. (1988) ont compilé les mentions de capture de salmonidés non indigènes effectuées dans toute l'étendue du fleuve Saint-Laurent, incluant l'estuaire et le Golfe du même nom. La présence de la truite brune a été signalée loin en aval des secteurs ensemencés, jusqu'à la limite des eaux douces, au voisinage de l'lle d'Orléans, de même que, toujours en eau douce, dans l'embouchure de deux tributaires de la Baie des Chaleurs (fig. 2).

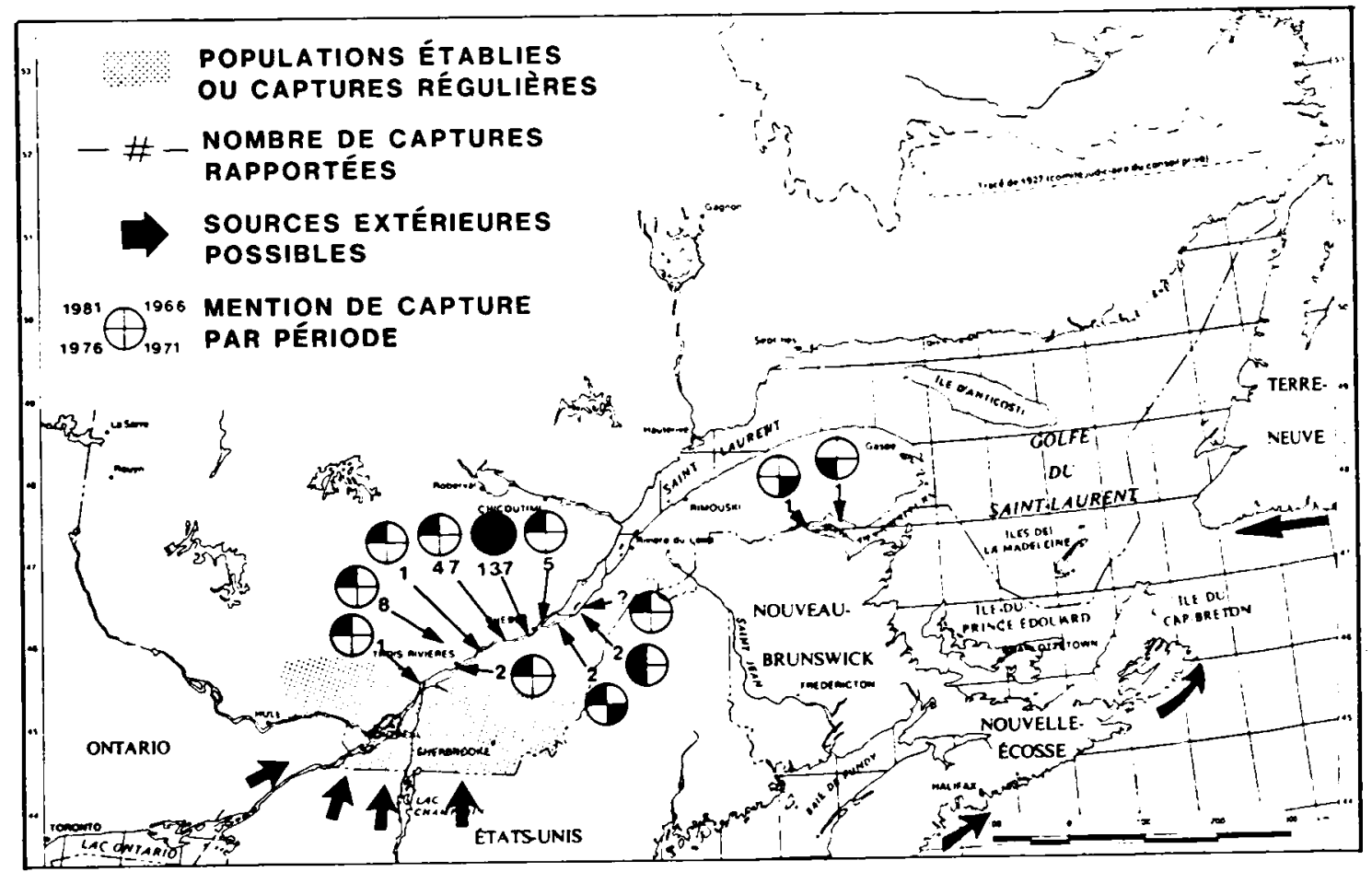

figure 2

: Aire de répartition de la truite brune dans la partie québécoise du bassin de drainage du fleuve Saint-Laurent pendant la période de 1966 à 1986 (adaplé de DUMONT of al., 1988).

figure 2 : Reported captures of brown trout in the Québec watershed of the Saint-Laurent River for the 1966-1986 period (modified from DUMONT of al., 1988).

Des captures de plus en plus nombreuses de truites brunes sont rapportées dans la pêcherie expérimentale de l'Aquarium du Québec, à Québec (ROBITAILLE et al., 1987). Notons que les spécimens capturés ne proviennent pas nécessairement de la plaine de Montréal; en fait des truites marquées et relâchées dans le lac Ontario (New York) sont capturées régulièrement à proximité de Montréal ou de Québec. La plupart des spécimens rapportés loin des sites d'ensemencement sont de grande taille. généralement supérieure à $400 \mathrm{~mm}$. D'autre part, à l'exception des captures enregistrées dans la Baie des Chaleurs, les déplacements connus actuellement ne présupposent pas nécessairement de migration en eau salée.

\subsection{Longévité, croissance et condition}

Les échantillons d'écailles disponibles révèlent l'atteinte d'un âge maximal similaire de 7 ans dans le fleuve Saint-Laurent $(n=71)$ et dans la rivière Richelieu $(n=8)$, et de quatre ans dans la rivière Châteauguay $(n=19)$. Les variations de la longueur totale en fonction de l'âge des truites brunes capturées dans le fleuve Saint-Laurent entre 1965 et 1977 ( $n=64$ ) apparaissent dans le Tableau I: ces truites mesurent en moyenne $296 \mathrm{~mm}$ à 2 ans, $492 \mathrm{~mm}$ à 4 ans et $553 \mathrm{~mm}$ à 6 ans. Par ailleurs, leur coefficient de condition de Fulton est de l'ordre de 1,28 ( $n=66$ ). 
Tableau 1 : Longueurs totales moyennes aux différents âges des truites brunes capturées dans le fleuve Saint-Laurent $(n=64)$.

Table I : Mean total lengths at age of brown trout caught in the Saint-Laurent River $(n=64)$.

\begin{tabular}{cccc}
\hline Groupe d'àge & Longueur totale & Nombre & Erreur a la moyenne \\
\hline 1 & 203,0 & 2 & \\
2 & 296,3 & 8 & 24,3 \\
3 & 405,9 & 10 & 18,3 \\
4 & 492,2 & 22 & 17,8 \\
5 & 527,4 & 14 & 22,4 \\
7 & 552,6 & 7 & 20,7 \\
\hline
\end{tabular}

\subsection{Reproduction}

La truite brune se reproduit dans les eaux de la plaine de Montréal. MONGEAU et al. (1980) rapportent des captures de fretins dans les rapides de Lachine. En outre, une vingtaine de sites de dépôt d'œufs ont été répertoriés récemment dans certains secteurs d'eau vive du fleuve SaintLaurent (rapides de Lachine, de Côteau-du-lac et de la Pointe du Buisson) (GUAY et COUILLARD, 1985 ; ENVIRONNEMENT ILLIMITÉ, 1987). Plusieurs autres sites de la région, non encore inventoriés, présentent également des conditions adéquates pour le dépôt des œufs.

Le déroulement de la fraye de la truite brune dans les rapides de Lachine, au cours de l'automne 1984, est décrit par GUAY et COUILLARD (1985). La ponte a eu lieu de la fin d'octobre à la fin de novembre et a été marquée par un pic d'activité durant la première quinzaine de novembre. La température de l'eau a graduellement décrû de $11,5^{\circ} \mathrm{C}$ à $3^{\circ} \mathrm{C}$ pendant l'ensemble de la période et de $10^{\circ} \mathrm{C}$ à $6,5^{\circ} \mathrm{C}$ lors du pic d'activité. Les vitesses de courant observées sur les sites de fraye ont varié de 0,31 à $1,25 \mathrm{~m} / \mathrm{s}$, et se sont généralement maintenues en deçà de $1,0 \mathrm{~m} / \mathrm{s}$. Les nids furent repérés à des profondeurs variant entre 30 et $100 \mathrm{~cm}$, et généralement en deçà de $50 \mathrm{~cm}$. La plupart d'entre eux étaient composés de gravier fin à moyen, comprenant parfois du sable, du gravier grossier et du galet, et formaient occasionnellement un monticule de quelques $\mathrm{cm}$ de hauteur. Des blocs, situés à proximité des nids, contribuaient probablement à l'accumulation du gravier.

\subsection{Contribution de la reproduction naturelle}

S'il est déjà prouvé que la truite brune se reproduit dans les eaux de la plaine de Montréal, il n'en reste pas moins que la contribution de la reproduction naturelle au soutien des populations du fleuve et de la rivière Richelieu paraît faible et peut-être même négligeable. DUMONT et DUMAS (1987), qui ont procédé à la compilation et à l'analyse des résultats de 2.980 excursions de pêche aux salmonidés dans la plaine de Montréal, entre 1983 et 1986, ont observé que l'abondance relative des truites brunes de moins de $30 \mathrm{~cm}$ (généralement des spécimens d'un an + ), dans la récolte sportive d'un groupe de pêcheurs volontaires, varie en fonction des quantités ensemencées annuellement.

En effet, la contribution des jeunes spécimens fut particulièrement faible en 1984 et 1986. alors que les quantités de truites ensemencees furent ou très limitées ou nulles (fig. 3 ). L'inverse fut noté en 1983 et 1985, alors que 36.000 truites furent ensemencées annuellement. En restreignant l'analyse aux sites pour lesquels ils disposaient d'un nombre élevé d'observations annuelles $(n>75)$, ces auteurs ont mis en évidence l'existence d'une relation statistique entre les densités de poissons ensemencés et la contribution des truites de petite taille (fig. 4). Trois points, où sont associées densités élevées et fortes contributions, sont principalement responsables de la tendance positive observée. Ces trois points représentent trois sites et deux années différentes d'ensemencement, 


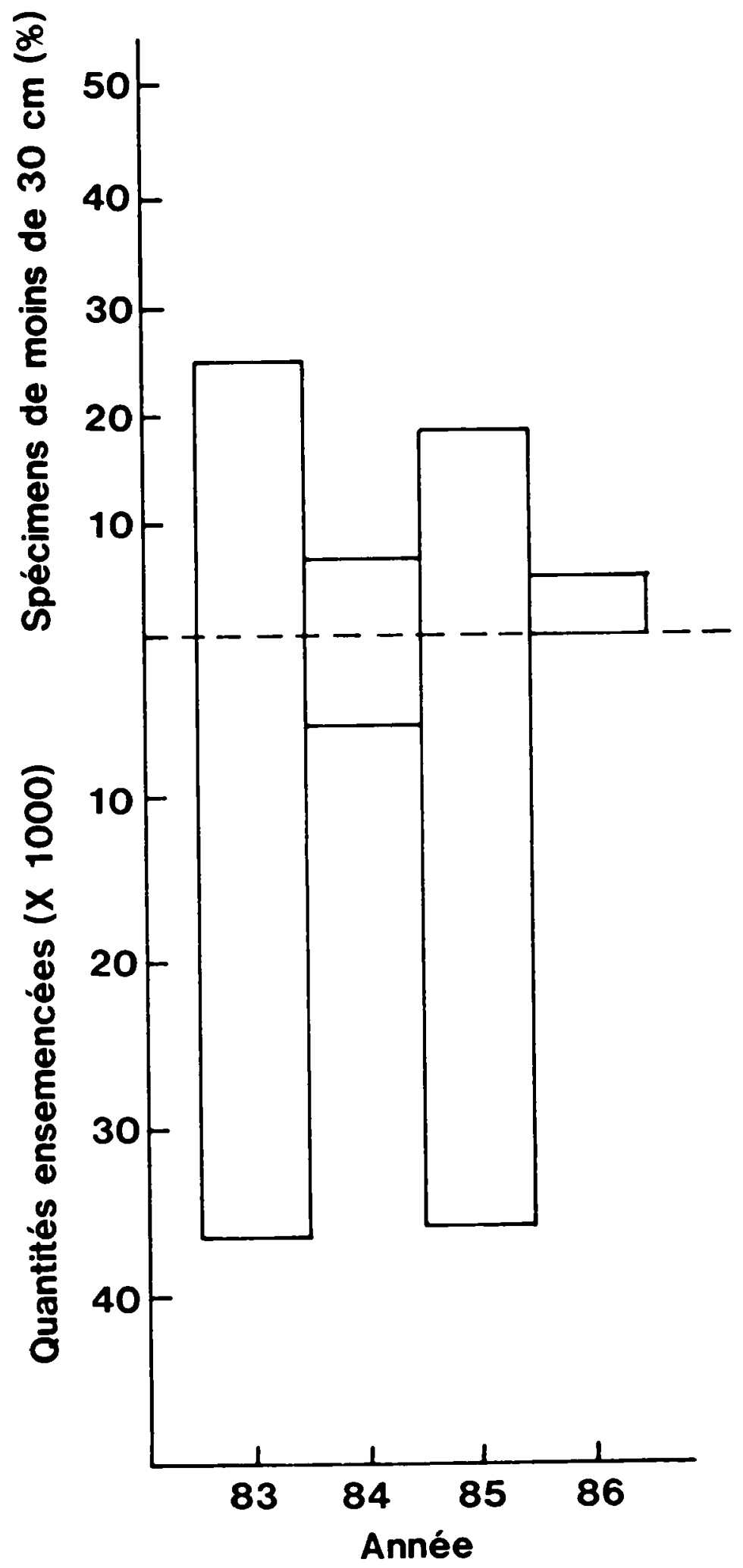

figure 3 : Contribution annuelle (en \%) des truites brunes de moins de $30 \mathrm{~cm}$ à la récolte des pêcheurs volontaires et quantités de truites d'un an + ensemencées de 1983 à 1986.

figure 3 : Annual contribution $(\%)$ of brown trout smaller than $30 \mathrm{~cm}$ to the sport fishing harvest of diary cooperators and number of yearling trout stocked during the period 1983 to 1986. 
suggérant ainsi que le phénomène n'est pas particulier à un site ou à une année donnée. Deux de ces trois sites figurent à chaque année parmi ceux répondant aux critères de sélection de l'analyse. Soulignons que les données en provenance de la rivière Châteauguay se sont avérées insuffisantes pour qu'elles puissent être prises en considération. La dispersion des points est importante et la relation peut être interprétée comme étant linéaire (équation 1) ou curvilinéaire (équation 2):

$$
\begin{aligned}
\text { contribution } \quad(\%)= & 2,4 \text { densité }-0,395 \\
& (r=0,75 ; p<0,005) \\
\text { log contribution }(\%)= & 1,063 \log \text { densité }+0,14 \\
& (r=0,73 ; p<0,005)
\end{aligned}
$$

Dans chacune des deux interprétations, l'ordonnée à l'origine n'est pas significativement différente de $0(p>0,05)$. En fait, en aucun cas. une faible densité d'ensemencement est associée à une forte contribution des truites de petite taille. phénomène qui aurait pu découler d'une contribution significative de la reproduction naturelle.

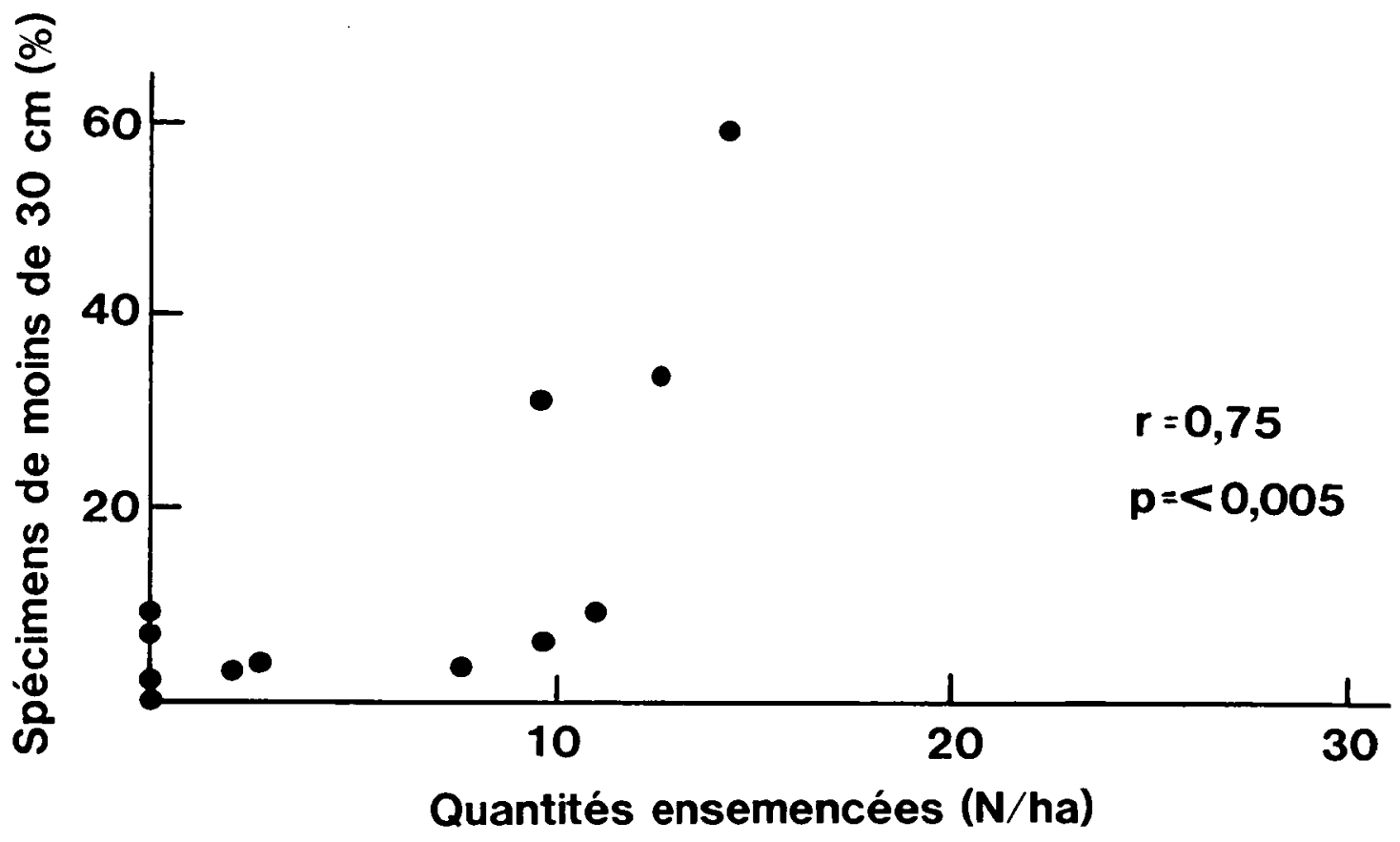

figure 4 : Relation entre la contribution des spécimens de moins de $30 \mathrm{~cm}$ à la récolte annuelle des pécheurs volontaires (\%) et les densités de truites d'un an + ensemencées (N/ha).

figure $4 \quad$ : Relation between the contribution of trout smaller than $30 \mathrm{~cm}$ to the sport fishing harvest of diary cooperators $(\%)$ and densities of yearling trout stocked (N/ha).

\subsection{Caractéristiques de la récolte sportive}

La taille des spécimens capturés à la ligne varie suivant les cours d'eau. Ceux du fleuve Saint-Laurent ont en moyenne $457 \mathrm{~mm}$ de longueur totale ( $\mathrm{n}=82$ ) et pèsent $1.400 \mathrm{~g}$; ceux de la rivière Richelieu, $437 \mathrm{~mm}(\mathrm{n}=83)$ et $1.100 \mathrm{~g}$, et ceux de la rivière Châteauguay, $310 \mathrm{~mm}(\mathrm{n}=313)$ et $500 \mathrm{~g}$. Les poids maximaux sont respectivement de $4.300 \mathrm{~g}, 2.600 \mathrm{~g}$ et $1.400 \mathrm{~g}$ (MONGEAU, LEGENDRE et al., 1979). 
Les pêcheurs ayant participé, entre 1983 et 1986, à lopération d'enregistrement de leurs captures de salmonidés ont rapporté 1.590 truites brunes, en 2.980 excursions, pour un succès moyen de 0.53 spécimen par excursion. D'après les déclarations de ces pêcheurs, la répartition des tailles des truites capturées varie considérablement d'un cours d'eau à l'autre. Dans le fleuve Saint-Laurent, $9,1 \%$ des captures ont moins de $30 \mathrm{~cm}$ de longueur et $28 \%$, plus de $50 \mathrm{~cm}$. Dans la rivière Richelieu, les chiffres correspondants sont $46,3 \%$ et $4,9 \%$, alors qu'ils sont de $70 \%$ et de $0 \%$ dans la rivière Châteauguay.

Toujours en se basant sur les mêmes déclarations, le succès de pêche subit d'importantes variations temporelles. Il est à son minimum au cours du mois d'août, augmente brusquement au cours de l'automne pour atteindre un sommet en décembre; il se maintient relativement élevé au cours de l'hiver et diminue graduellement par la suite (fig. 5). C'est au cours de l'hiver que le potentiel de capture des gros spécimens, de $50 \mathrm{~cm}$ et plus, est le plus élevé. Ainsi, en janvier et février, plus de $60 \%$ des captures appartiennent à cette catégorie. Leur contribution à la récolte devient minimale en août $(12,5 \%)$ et décembre $(9 \%)$ et demeure relativement faible et stable au cours des autres mois (17 à $35 \%$ ).
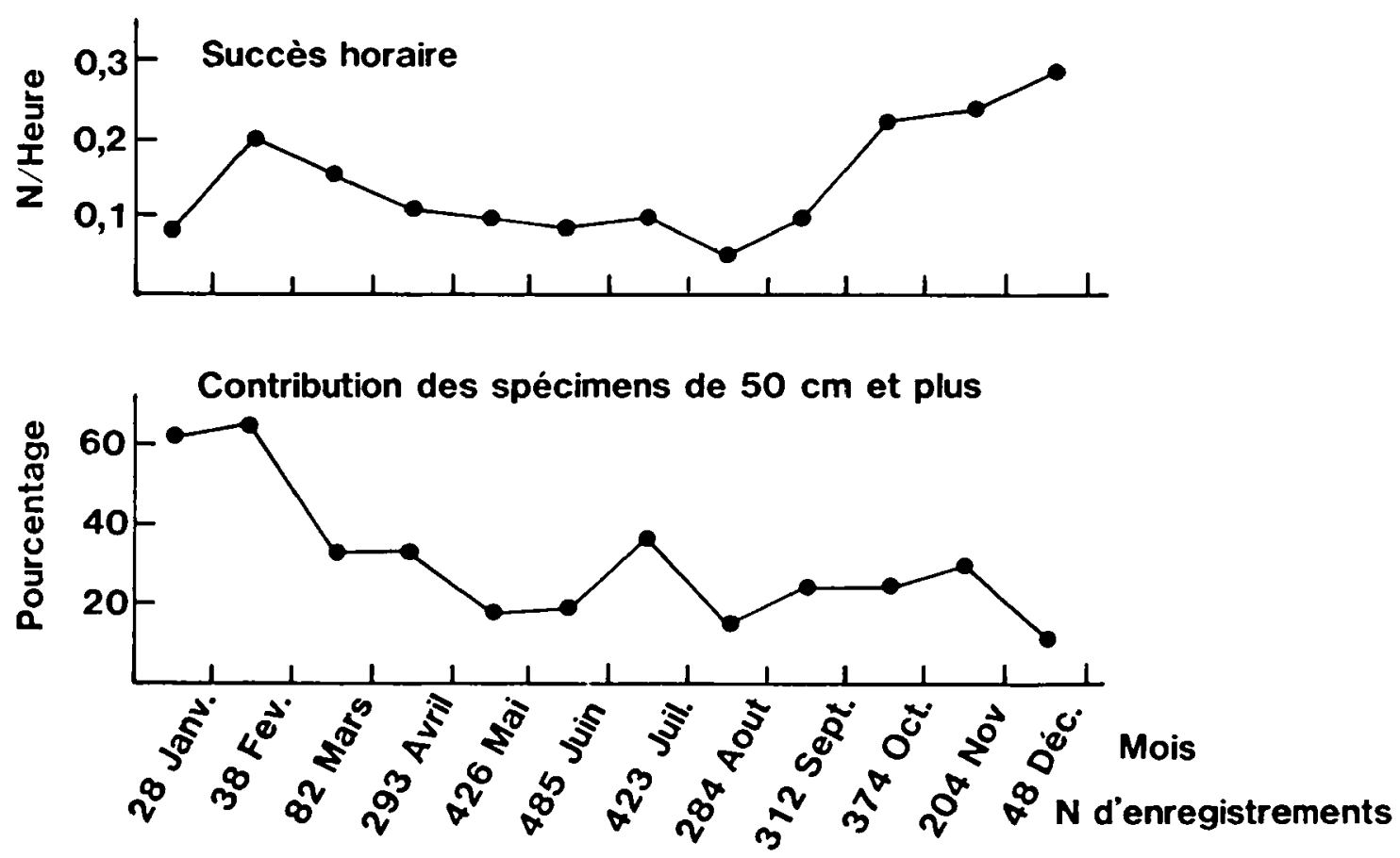

figure 5

: Variations mensuelles du succès horaire (nombre de truites par heure de pêche) et de la contribution des truites de plus de $50 \mathrm{~cm}(\%)$ à la récolte des pécheurs
volontaires, de 1983 à 1986 .

figure 5 : Monthly variations of the mean catch per hour of sport fishing and of the contribution of trout larger than $50 \mathrm{~cm}(\%)$ to the harvest of diary cooperators, from 1983 to 1986.

\section{DISCUSSION ET CONCLUSION}

\subsection{La truite brune}

Les truites brunes ensemencées dans la plaine de Montréal paraissent s'être bien adaptées à un milieu qu'on pourrait qualifier de marginal pour un salmonidé. RALEIGH et al. (1986) sont d'avis que l'habitat de cette espèce est caractérisé par des eaux fraîches ou froides. NEEDHAM (1969) mentionne qu'au-delà de $27,2^{\circ} \mathrm{C}$, la viabilité d'une population de truite brune ne peut pas être assurée. D'après RALEIGH et al., la gamme des températures tolérées par l'espèce s'étendrait entre 0 et $27^{\circ} \mathrm{C}$. La truite brune a cependant des exigences différentes au cours de son cycle vital. Ainsi, la 
température optimale pour la croissance et l'alimentation des juvéniles et des adultes varierait entre 12 et $19^{\circ} \mathrm{C}$ (FROST et BROWN, 1967 ; MILLS, 1971 ; BROWN, 1973 ; TEBO, 1975) ; pour les fretins, elle oscillerait entre 7 et $15^{\circ} \mathrm{C}$ (BROWN, 1973) alors que les alevins, elle varierait entre 2 et $13^{\circ} \mathrm{C}$ (MARKUS, 1962 ; FROST et BROWN, 1967). SPAAS (1960) situe la température létale moyenne à $25,46{ }^{\circ} \mathrm{C}$ dans le cas de l'alevin, à $28,5^{\circ} \mathrm{C}$ dans celui de la truite brune d'un an + , et à $29^{\circ} \mathrm{C}$ dans celui de l'adulte. En été, les eaux du fleuve Saint-Laurent et de la rivière Richelieu atteignent généralement des températures de 23 ou $24^{\circ} \mathrm{C}$ et dépassent parfois ces maximums de 1 ou $2^{\circ} \mathrm{C}$. La marge de sécurité quant à la survie de l'espèce, et tout particulièrement des jeunes individus, est donc très faible et même inexistante en certaines occasions. C'est sans doute en se confinant dans les eaux rapides et turbulentes, caractérisées par une forte évaporation et une oxygénation efficace, que la truite brune parvient à subsister au cours de la période estivale. Dans la plaine de Montréal, ces milieux totalisent une superficie d'une trentaine de $\mathrm{km}^{2}$. concentrée principalement dans deux sites et ne représentant qu'une très faible proportion de l'ensemble des eaux de la région.

Bien que confinée dans des habitats marginaux, la truite brune se développe rapidement dans les eaux du Saint-Laurent. Son taux de croissance dépasse celui des truites du sud de l'Ontario rapporté par MANSELL (1966) et se compare avantageusement aux valeurs les plus élevées, citées par CARLANDER (1969), en provenance de rivières américaines et françaises (Tableau II). De même, le coefficient de condition mesuré pour le fleuve, soit 1,28 , se situe au centre de la gamme đe ceux rapportés par CARLANDER (1969), lesquels s'étalent entre 0,74 et 1,74.

Tableau II : Croissance comparée de la truite brune. Les longueurs moyennes rapportées par CARLANDER (1969) pour des populations européennes et nord-américaines correspondent aux valeurs maximales répertoriées.

Table II : Comparative growth of brown trout. Mean total lengths reported by CARLANDER (1969) for european and american populations are maximal values observed.

\begin{tabular}{|c|c|c|c|c|c|c|c|c|}
\hline \multirow{2}{*}{ SITE } & \multicolumn{8}{|c|}{ Longueur totale à l'âge (mm) } \\
\hline & 1 & 2 & 3 & 4 & 5 & 6 & 7 & 8 \\
\hline $\begin{array}{c}\text { Fleuve Saint-Laurent } \\
\text { (cette étude) }\end{array}$ & 203 & 296 & 406 & 492 & 527 & 553 & 571 & \\
\hline $\begin{array}{l}\text { Sud de l'ontario } \\
\text { (Mansell. 1966) }\end{array}$ & 165 & 249 & 388 & 427 & & & & \\
\hline $\begin{array}{l}\text { Rivières de France } \\
\text { (Carlander, 1969) }\end{array}$ & 109 & 211 & 290 & 371 & 439 & 330 & & \\
\hline $\begin{array}{l}\text { Rivières américaines } \\
\text { (Carlander, 1969) }\end{array}$ & 152 & 224 & 335 & 404 & 572 & 513 & 648 & 703 \\
\hline
\end{tabular}

L'analyse des déclarations d'un groupe de pêcheurs sportifs suggère le fait que les ensemencements contribuent significativement à la récolte. La relation statistique mise en évidence entre les densités de truites ensemencées et la contribution des truites de petite taille à la récolte peut s'adapter à différents modèles. Suivant la première hypothèse (linéarité, équation 1), chaque poisson ensemencé par hectare entraîne, en moyenne, un accroissement de la contribution des individus de petite taille à la récolte de l'ordre de 2,4\%. La seconde hypothèse (curvilinéarité, équation 2) suggè re que les densités d'ensemencement élevées se traduisent par des contributions proportionnellement beaucoup plus fortes que dans le cas des densités faibles. Au-delà d'environ 10 spécimens par hectare, la contribution des spécimens de petite taille augmenterait de façon exponentielle. Les causes d'une telle tendance paraissent cependant difficilement imaginables à moins de supposer qu'il se produirait un effet de saturation à des densités très faibles, hypothèse qui nous paraît improbable. 
Toutefois, que la relation soit du type linéaire ou curvilinéaire, l'ordonnée à l'origine ne peut pas être considérée comme étant significativement différente de zéro. Il est donc impossible d'écarter l'hypothèse à l'effet que, en l'absence d'ensemencement, la contribution des plus petits spécimens à la récolte de 1983 à 1986 ait été nulle ou, en d'autres mots, que l'influence de la reproduction naturelle, dont l'existence a par ailleurs été démontrée, ait été faible ou négligeable. A notre avis, cette observation est valable dans le cas de la plupart des sites du fleuve Saint-Laurent et de la rivière Richelieu, même si, dans les faits, des truites de moins de $30 \mathrm{~cm}$ furent enregistrées en 1986, alors qu'il n'y avait pas eu d'ensemencement. En effet, le taux de croissance individuelle étant variable, l'indice de taille retenu (moins de $30 \mathrm{~cm}$ ) peut également inclure des spécimens de deux ans. D'autre part, des apports venus de l'extérieur sont toujours possibles du fait que des quantités considérables de truites brunes sont ensemencées annuellement dans la partie est du lac Ontario (fleuve Saint-Laurent) et dans le lac Champlain (rivière Richelieu).

Même si la truite brune se reproduit dans les eaux de la plaine de Montréal, il est possible que le frai ne rencontre pas les conditions favorables à son développement. Ainsi, on sait que les alevins sont encore plus sensibles aux températures élevées que les truites plus âgées (SPAAS, 1960). C'est peut-être pourquoi, dans les années 1970, l'utilisation d'alevins et de fretins a avantageusement cédé la place à celle des truites d'un an . Il y a également lieu de croire que, dans les eaux de la plaine du Saint-Laurent, les alevins et les fretins de truites brunes subissent une prédation excessive. Cette contrainte pourrait être particulièrement sévère dans le fleuve Saint-Laurent et dans la rivière Richelieu. Quant à la rivière Châteauguay, qui n'a pas été retenue parmi les sites soumis à l'analyse, la situation est sans doute différente. En effet, la section ensemencée est plus favorable à la survie des jeunes de l'année, tant au point de vue de la température de l'eau que de la compétition interspécifique.

Pour ce qui est du pêcheur sportif de la plaine de Montréal, l'introduction de la truite brune constitue un avantage important. Depuis les premiers ensemencements, les pêcheurs se sont adaptés graduellement aux techniques particulières de la pêche aux salmonidés, de sorte que présentement leur succès se compare à celui des pêcheurs de l'arrière-pays pour les espèces indigènes. En outre, principalement dans les grands cours d'eau comme le fleuve Saint-Laurent, la taille des captures est généralement imposante.

\subsection{La truite brune et la truite arc-en-ciel}

Depuis le début des ensemencements de truites brunes dans les eaux de la plaine de Montréal en 1965, un effort parallèle a également été déployé en faveur de la truite arc-en-ciel (Oncorhynchus mykiss). Cependant, même si la truite brune a généralement été ensemencée en quantité équivalente ou inférieure à celle de sa congénère, son rendement à la pêche à la ligne a constamment été supérieur. Ainsi, MONGEAU, LEGENDRE et al. (1979) rapportent que pour des quantités relativement similaires de fretins (163.500 truites arc-en-ciel par rapport à 287.500 truites brunes) et d'individus d'un an - (62.700 par rapport à 76.590) ensemencés entre 1970 et 1975 . les pêcheurs ont rapporté 335 captures de truites arc-en-ciel, ce qui est 2,4 fois moindre que les 805 mentions correspondantes de truites brunes. De même, DUMONT et DUMAS (1987) constatent que les pêcheurs ayant participé à l'opération d'enregistrement de leurs captures de salmonidés, entre 1983 et 1986, ont récolté 1.095 truites arc-en-ciel contre 1.590 truites brunes, alors que, entre 1982 et 1986 , les quantités de truites arc-en-ciel d'un an + ensemencées avaient été 1,8 fois plus importantes. Cette dominance est manifeste dans tous les sites, excepté ceux de la rivière Richelieu.

Cette plus grande disponibilite de la truite brune est d'autant plus surprenante que l'espèce est généralement moins vulnérable aux engins de pêche sportive (COOPER, 1959). Plusieurs facteurs peuvent expliquer ce phénomène. D'après les données colligées par MONGEAU. LEGENDRE et al. (1979), il appert que, dans la région de Montréal, la truite arc-en-ciel vit moins longtemps que la truite brune. L'àge maximal des spécimens rapportés par les pécheurs sportifs ne dépasse pas 4 ans. D'autre part. CRESSWELL (1981) rapporte que la truite arc-en-ciel manifeste un comportement migrateur plus accentué que celui de la truite brune, laquelle a plutôt tendance à demeurer au voisinage des sites d'ensemencement. MONGEAU et BRISEBOIS (1982) ont confirmé ces conclusions. Aucune des recaptures de truites brunes étiquetées n'a été rapportée à l'extérieur de la région de Montréal alors que 21 des 570 recaptures de truites arc-en-ciel l'ont été en aval de cette région. Une de ces truites avait même atteint Trois-Pistoles, à $500 \mathrm{~km}$ en aval. En outre, DUMONT et al. (1988) ont relevé plusieurs mentions de truites arc-en-ciel dans les tributaires de l'estuaire et du Golfe du Saint-Laurent alors que celles de truites brunes étaient pour la plupart limitées aux eaux douces ou saumâtres du fleuve Saint-Laurent, en amont de l'île d'Orléans.

Bien que l'on concède généralement un léger avantage à la truite brune en ce qui concerne sa tolérance aux températures élevées, cette hypothèse ne serait valable que dans le cas du fleuve Saint-Laurent. En effet, elle ne tient pas dans la portion ensemencée de la rivière Châteauguay dont 
la température estivale se maintient en-deçà de $25^{\circ} \mathrm{C}$ (LEGENDRE et al., 1980) et où la truite brune domine les captures même si les quantités de truites arc-en-ciel ensemencées y furent nettement supérieures. En outre, la truite arc-en-ciel semble mieux réussir que la truite brune dans la rivière Richelieu, en dépit du fait que les eaux de cette rivière atteignent une température supérieure à celle du fleuve durant l'été.

La contribution de la truite brune au rendement de la pêche s'avère également supérieure à celle de la truite arc-en-ciel par la taille des captures. C'est ainsi que la proportion de spécimens de $500 \mathrm{~mm}$ et plus, dans la récolte des pêcheurs volontaires, est de $25,6 \%$ pour la première espèce comparativement à $8,8 \%$ pour la seconde (DUMONT et DUMAS, 1987).

\subsection{Problèmes posés par l'introduction de la truite brune}

La truite brune n'est que l'un des nombreux salmonidés non indigènes qui ont été introduits dans le bassin de l'Atlantique. En effet, la truite arc-en-ciel et au moins quatre espèces de saumons du Pacifique font l'objet d'ensemencements massifs dans l'est de l'Amérique du Nord. En fait, comparativement aux provinces et états voisins, les déversements québécois sont très modestes. La présence de ces salmonidés non indigènes dans la système hydrographique du Saint-Laurent a eu un impact positif sur la pêche sportive ainsi que sur l'image projetée par ces cours d'eau. Le fait que des truites et des saumons puissent y survivre et s'y développer a contribué à leur revalorisation et à faire prendre conscience à la population québécoise de la nécessité de se soucier de leur réhabilitation. Par contre, ces introductions inquiètent les aménagistes. DUMONT et al. (1988) ont fait le point sur l'extension d'aire de ces poissons au Québec et sur les impacts possibles de ces essais d'implantation réalisés sans évaluation préalable.

Ces poissons, élevés en pisciculture, peuvent être porteurs de maladies susceptibles d'être transmises indistinctement aux populations vivant en aval ou en amont des sites de déversement. Plusieurs espèces indigènes peuvent être affectées mais, à ce jour, c'est le saumon atlantique (Salmo salar) qui a retenu l'attention des aménagistes (SINDERMANN, 1986). Les risques de modification du matériel génétique ont également été évoqués. Des cas d'hybridation naturelle avec des salmonidés indigènes ont déjà été rapportés: S. trutta X Salvelinus fontinalis (WITZEL, 1983), S. trutta X S. salar (BELAND et al., 1981; VERSPOOR, 1988). La fréquence du dernier type d'hybrides dans les eaux à l'est de Terre-Neuve $(0,9 \%)$ est trois fois supérieure à celle mesurée dans les eaux des lles britanniques où les deux espèces sont indigènes (VERSPOOR, 1988).

La compétition interspécifique est également mentionnée. Les risques sont très restreints dans la plaine de Montréal puisque les salmonidés y sont confinés à des habitats de superficie limitée. Il en est de même dans l'arrière-pays où une législation limite le choix des espèces à ensemencer à quelques salmonidés indigènes (VEZZINA, 1986). Par contre, ce sont les introductions dans le système hydrographique du Saint-Laurent, ouvert sur les Grands Lacs et sur l'Atlantique, qui suscitent le plus d'inquiétudes. L'éventuelle implantation de salmonidés non indigènes dans les tributaires de l'estuaire et du Golfe du Saint-Laurent, caractérisés par une faible productivité et une faible diversité, est probable. Or, ces derniers sont susceptibles d'y entrer en interaction avec les peuplements anadromes et dulcicoles de saumon atlantique, d'omble de fontaine et d'omble chevalier (Salvelinus salvelinus) (DUMONT et al., 1988). Cependant, la truite brune n'est pas l'espèce la plus menaçante. En Europe, où elle cohabite avec le saumon atlantique dans certains cours d'eau, les deux espèces ne s'alimentent des mêmes proies qu'en été, lorsque la nourriture est abondante (PEDLEY et JONES, 1978). Néanmoins, il lui est arrivé de déplacer ou même d'éliminer l'Omble de fontaine dans certains cours d'eau de l'Amérique du Nord (FAUSCH et WHITE, 1981 ; WATERS, 1983).

Les performances supérieures de la truite brune par rapport à la truite arc-en-ciel nous ont amenés, au cours des dernières années, à augmenter nos ensemencements de la première et à réduire ceux de la seconde. Par ailleurs, afin de réduire les risques de propagation et, par la même occasion, de profiter d'un potentiel de croissance plus élevé, les prochains ensemencements de salmonidés non indigènes (truites brunes et arc-en-ciel) dans les eaux de la plaine de Montréal devraient être réalisés au moyen d'individus stériles.

\section{REMERCIEMENTS}

Notre reconnaissance s'adresse d'abord aux nombreux pêcheurs sportifs de la plaine de Montréal pour leur collaboration. Nous tenons également à remercier Monsieur V. LEGENDRE pour la lecture des écailles de truite brune, Madame J. BRISEBOIS ainsi que Messieurs G. ROY, $M$. BEAUDOIN et B. DUMAS pour leur contribution à la collecte et à la compilation des données, de même que Monsieur P.A. ROY pour la réalisation des figures et Mesdames F. ROCHEFORT et D. JACQUES pour le traitement du texte. 


\section{BIBLIOGRAPHIE}

ANONYME, 1987. La pêche récréative au Québec en 1985. Ministère des Pêches et Océans et Ministère du Loisir, de la Chasse et de la Pêche, $16 \mathrm{p}$.

BELAND K. F., ROBERTS F.L. and SAUNDERS R. L., 1981. Evidence of Salmo salar X Salmo trutta hybridization in a North American river. Can. J. Fish. Aquat. Sci., 38, 552-554.

BÉRUBÉ R., 1983. Aménagement d'une centrale au barrage Chambly. Description du milieu naturel. Rapport synthèse, Hydro-Québec, Vice-Présidence Environnement, Écologie bio-physique, 161 p. et annexes.

BROWN H. W., 1973. A literature review and evaluation of the effects of temperature upon the fish of the New River Drainage in Giles County, Virginia. Am. Electric Power Serv. Corp. (Draft.).

CARLANDER K. D.. 1969. Handbook of freshwater fishery biology. Vol. 1. The lowa State University Press, Ames, lowa, $752 \mathrm{p}$.

COOPER P., 1959. Trout stocking as an aid to fish management. Penn. St. Univ., College of Agriculture, Bull. 663, 21 p.

COTÉ M., 1983. Études de la répartition géographique des espèces de poissons d'eau douce non indigènes au Québec. Le groupe Teknika Urbanitek Inc. pour le Ministère du Loisir, de la Chasse et de la Pêche, 36 p. + 1 annexe.

CRESSWELL R. C., 1981. Post-stocking movements and recapture of hatchery-reared trout released into flowing waters-a review. J. Fish Biol., 18, 429-442.

DUMONT P., BERGERON J. F., DULUDE P., MAILHOT Y., ROULEAU A., OUELLET G. and LEBEL J.P., 1988. Introduced Salmonids: Where are they going in Quebec watershed of the Saint-Laurent River? Fisheries, 13 (3), 9-17.

DUMONT P. et DUMAS B., 1987. Les ensemencements de Salmonidés dans les eaux de la plaine de Montréal: un essai d'évaluation de leur efficacité par le biais des carnets de pêcheurs. Québec, Ministère du Loisir, de la Chasse et de la Pêche, Service de l'aménagement et de l'exploitation de la faune, Montréal, Rap. Trav. 06-01, $41 \mathrm{p}$.

ENVIRONNEMENT ILLIMITÉ, 1987. Révision du mode d'exploitation des ouvrages compensateurs du fleuve Saint-Laurent de Côteau à Pointe-des-Cascades, pour le Service Encadrements et Conseils, Direction Environnement, Hydro-Québec, 196 p. + annexes et cartes.

FAUSCH K. D. and WHITE R. J., 1981. Competition between brook trout (Salvelinus fontinalis) and brown trout (Salmo trutta) for positions in a Michigan stream. Can J. Fish. Aquat. Sci., 38, 1220-1227.

FROST W.E. and BROWN M. E., 1967. The trout. Collins, St. James' Place, London, 286 p.

GUAY G. et COUILLARD M., 1985. Etude de l'utilisation printanière et automnale des rapides de Lachine par les poissons. Projet Archipel de Montréal (1984). Pour le Secrétariat Archipel, XVII + 156 p. +5 annexes.

LECLERC J., 1984. Marquage et repérage radiotélémétriques de 11 espèces de poissons du territoire central du projet Archipel entre octobre 1982 et août 1983. Synthèse des résultats. Québec, Ministère du Loisir, de la Chasse et de la Pêche, Service Archipel, Rap. tech. 20, $164 p$.

LEGENDRE V., MONGEAU J.-R., LECLERC J. et BRISEBOIS J., 1980. Les salmonidés des eaux de la plaine de Montréal. 1. Historique, 1534-1977. Québec, Ministère du Loisir, de la Chasse et de la Pêche, Service de l'Aménagement et de l'Exploitation de la Faune, Montréal, Rapp. tech. 06-27, $280 p$.

MACCRIMMON H. R. and MARSHALL T.L., 1968. World distribution of brown trout, Salmo trutta. J. Fish. Res. Board Can., 25, 2527-2548.

MACCRIMMON H.R., MARSHALL T. L. and GOTS B.L., 1970. World distribution of brown trout, (Salmo trutta): further observations. J. Fish. Res. Board Can., 27, 811-818.

MANSELL W. O., 1966. Brown trout in southwestern Ontario. Ontario Fish Wildl. Rev. 5 (2), 3-8.

MARKUS H. C., 1962. Hatchery-reared Atlantic salmon smolts in ten months. Prog. Fish-Cult., 24, $127-130$.

MILLS D., 1971. Saimon and trout: A ressource, its ecology, conservation and management. St. Martin's Press, New York, 351 p.

MONGEAU J.-R., 1975. L'implantation de la Truite brune, Salmo trutta, dans les eaux de la plaine de Montréal. Québec, Ministère du Loisir, de la Chasse et de la Pêche, Service de l'aménagement et de l'exploitation de la faune, Montréal, Rapp. tech. 06-05, 43 p.

MONGEAU J.-R., 1979. Dossier des poissons du bassin versant de la Baie Missisquoi et de la rivière Richelieu, 1954 à 1977. Québec, Ministère du Loisir, de la Chasse et de la Pêche, Service de 
l'aménagement et de l'exploitation de la faune, Montréal, Rapp. tech. 06-24, 251 p.

MONGEAU J.-R. et BRISEBOIS J., 1982. Ce qu'il advient des truites ensemencées dans les eaux de la plaine de Montréal, d'après les expériences d'étiquetage et de recapture, de 1977 à 1981 inclusivement. Québec, Ministère du Loisir, de la Chasse et de la Pêche, Service de l'Aménagement et de l'Exploitation de la Faune, Montréal, Rapp. tech. 06-35, 47 p.

MONGEAU J.-R., LECLERC J. et BRISEBOIS J., 1979. Les poissons du bassin de drainage de la rivière Châteauguay, leur milieu naturel, leur répartition géographique et leur abondance relative. Québec, Min. du Tourisme, de la Chasse et de la Pêche, Service de l'aménagement et de l'exploitation de la faune, Montréal, Rapport technique 06-23, $105 \mathrm{p}$.

MONGEAU J.-R., LECLERC J. et BRISEBOIS J., 1980. La répartition géographique des poissons, les ensemencements, la pêche sportive et commerciale, les frayères et la bathymétrie du fleuve Saint-Laurent dans le Bassin de La Prairie et les Rapides de Lachine. Québec, Ministère du Loisir, de la Chasse et de la Pêche, Service de l'Aménagement et de l'Exploitation de la Faune, Montréal, Rapp. tech. 06-29, 145 p.

MONGEAU J.-R., LEGENDRE V., LECLERC J. et BRISEBOIS J., 1979. Les salmonidés des eaux de la plaine de Montréal. 2. Biométrie, biogéographie, 1970-1975, et registre des pêches, 1941-1976. Québec, Ministère du Loisir, de la Chasse et de la Pêche, Service de l'Aménagement et de l'Exploitation de la Faune, Montréal, Rapp. tech. 06-28, 139 p.

MONGEAU J.-R. et MASSÉ G., 1976. Les poissons de la région de Montréal, la pêche sportive et commerciale, les ensemencements, les frayères, la contamination par le mercure et les PCB. Québec, Min. du Tourisme, de la Chasse et de la Pêche, Service de l'Aménagement et de l'Exploitation de la Faune, Montréal, Rapport technique 06-13, $286 \mathrm{p}$.

NEEDHAM P. R., 1969. Trout streams (revised edition - C.F. Bond) Winchester Press, New York, $241 \mathrm{p}$.

PEDLEY R. B. and JONES J. W., 1978. The comparative feeding behavior of brown trout (Salmo trutta L.) and Atlantic salmon (Salmo salar L.) in Llyn Dwythwch, Wales. J. Fish Biol., 12, 239-256.

RALEIGH R. F., ZUCKERMAN L. D. and NELSON P. C., 1986. Habitat suitability index models and instream flow suitability curves: Brown trout, revised. Us. Fish Wildl. Serv. Biol. Rep. 82 (10.124), 65 p. (First printed as: FWS/OBS-82/10.71, September 1984).

RICKER W. E., 1971. Methods for assessment of fish production in fresh waters. IBP Handbook $N^{\circ}$ 3. Blackwell scientific publications, Oxford and Edinburgh, $348 \mathrm{p}$.

ROBITAILLE J. A., POMERLEAU C. et PAULHUS P. J., 1987. Analyse sommaire des captures de la pêcherie expérimentale de l'Aquarium du Québec, de 1971 à 1986. Ministère du Loisir, de la Chasse et de la Pêche du Québec, Direction de la faune aquatique et Direction régionale de Québec. Rapp. tech. 87-02, VI + 54 p.

SINDERMANN C.J., 1986. Strategies for reducing risks from introductions of aquatic organisms : a marine perspective. Fisheries, 11 (2), 10-15.

SPAAS J. T., 1960. Contribution to the comparative physiology and genetics of the European salmonidae III. Temperature resistance of different ages. Hydrobiologia, 15, 78-88.

TEBO L. B. Jr., 1975. Review of selected parameters of trout stream quality. Pages 20-32 in Symposium on trout habitat research and management proceedings. U.S. For. Serv., Southeast. For. Exp. Stn., Asneville, NC.

VERSPOOR E., 1988. Widespread hybridization between native Atlantic salmon, Salmo salar, and introduced brown trout, S. trutta, in eastern Newfoundland. J. Fish. Biol., 32, 327-334.

VEZINA R., 1986. Regulations and policies on fish introduction in the province of Quebec. American Fisheries Society, Atlantic International chapter, 12th annual meeting, Sept. 21-23th Magog, Quebec, $16 p$.

WATERS T. F., 1983. Replacement of brook trout by brown trout over 15 years in a Minnesota stream: production and abundance. Trans. Am. Fish. Soc., 112, 137-146.

WITZEL L. D., 1983. The occurrence and origin of tiger trout (Salmo trutta X Salvelinus fontinalis), in Ontario streams. Can. Field Nat., 97, 99-102. 